\title{
Effect of miR-1244 on cisplatin-treated non-small cell lung cancer via MEF2D expression
}

\author{
GUANG-JIAN LI, GUANG-QIANG ZHAO, JIA-PENG YANG, YONG-CHUN ZHOU, \\ KAI-YUN YANG, YU-JIE LEI and YUN-CHAO HUANG
}

\begin{abstract}
Department of Thoracic Surgery, Tumor Hospital of Yunnan Province, The Third Affiliated Hospital of Kunming Medical University, Kunming, Yunnan 650118, P.R. China
\end{abstract}

Received June 26, 2016; Accepted August 6, 2016

DOI: $10.3892 /$ or.2017.5624

\begin{abstract}
The aim of this study was to investigate the function of miR-1244 in cisplatin-treated non-small cell lung cancer (NSCLC). The results of quantitative PCR analysis revealed that the expression levels of miR-1244 in cisplatin-treated A549 and NCI-H522 human lung cancer cell lines were lower than those in untreated A549 and NCI-H522 cells. Similarly, the expression level of miR-1244 in NSCLC tissue samples from cisplatin-treated patients was also lower than that in non-cisplatin-treated NSCLC patients. Notably, the overall survival times of cisplatin-treated NSCLC patients with high miR-1244 expression were superior to those patients with low miR-1244 expression. We found that overexpression of miR-1244 suppressed cell viability and increased LDH toxicity in cisplatin-treated A549 and NCI-H522 cells. Additionally, overexpression of miR-1244 induced the apoptosis of cisplatin-treated A549 and NCI-H522 cells. Furthermore, overexpression of miR-1244 promoted caspase-3 activity and p53 and Bax protein expression, and suppressed myocyte enhancer factor 2D (MEF2D) and cyclin D1 protein expression in cisplatin-treated A549 and NCI-H522 cells. Small interfering RNA (siRNA) targeting MEF2D suppressed the protein expression of MEF2D, and was able to decrease the proliferation, promote caspase-3 activity, p53 and Bax protein expression and inhibit cyclin D1 protein expression in cisplatin-treated A549 and NCI-H522 cells following the overexpression of miR-1244. In summary, we found that miR-1244 affected cisplatin-treated NSCLC via MEF2D expression.
\end{abstract}

\section{Introduction}

Non-small cell lung cancer (NSCLC) is a malignant tumor with one of the highest morbidity rates worldwide, and is the

Correspondence to: Dr Yun-Chao Huang, Department of Thoracic Surgery, Tumor Hospital of Yunnan Province, The Third Affiliated Hospital of Kunming Medical University, 519 Kunzhou Road, Kunming, Yunnan 650118, P.R. China

E-mail: huangyunchaokm@163.com

Key words: miR-1244, non-small cell lung cancer, MEF2D, Bax most common cause of malignant tumor-related death (1). With the improvements in living standards and health, the morbidity of NSCLC has been decreased in recent years (2). However, its morbidity in male patients still ranks second out of all types of malignant tumors. Furthermore, the incidence of NSCLC appears to be rising in females (3). Although the treatment of NSCLC has been constantly improving, the survival rate has not increased substantially. This may be due to common late diagnosis, late treatment and complexity (4). Therefore, it is necessary to explore the pathogenetic mechanisms underlying NSCLC and the potential modulatory mechanisms involved to facilitate its early diagnosis and early treatment.

In recent years, molecular diagnosis and targeted therapies have provided a new approach to the integrated control of NSCLC (5). Individual gene expression patterns, individual neoplasm staging and their relevance to patient prognosis have gradually become an area of interest in NSCLC research (6). The pathogenetic mechanism of NSCLC is associated with frequent mutation, amplification and epigenetic changes of tumor-related genes (7). Epigenetic alterations do not affect genomic sequence, but can cause protein expression changes that result in tumor development and progression (7). Studies of epigenetics mainly focus on DNA methylation, chromatin rearrangement, and changes to RNA editing. Furthermore, miRNAs are currently a hotspot for research into tumor-related changes $(5,6)$. Recent research indicates that alterations to the regulation of miRNAs may to some extent contribute to the occurrence of malignant tumors.

miRNAs are small non-coding RNA molecules that are processed by specific incision enzymes; transcriptional precursors consisting of double-stranded RNA (pri-miRNAs) are cleaved by Drosha and Dicer enzymes (8). miRNAs have complementary sequence to their target mRNA and base pair with the target sequence resulting in mRNA degradation or decreased translation, inducing gene silencing (9). Gene expression is negatively regulated by the interaction of a miRNA with the 3 ' non-coding region of the target gene mRNA. This regulation of protein translation by miRNAs influences a wide variety of physiological and abnormal cell processes (10). miRNAs are also highly specific; as biomarkers, they may have an important role in tumor prevention (11). In NSCLC, miRNAs have been shown to affect tumor formation, occurrence and development (11). Although in many cases the 
precise functions of specific miRNAs are not clear, miRNAs are regarded as important contributors to various physiological and developmental processes (12).

Myocyte enhancer factor 2D (MEF2D) is a gene of the MEF2 family, and contains a structural domain of the regulatory factor MCM1 (13). Its main function is to regulate the survival and differentiation of multiple cell types. Early studies on MEF2 were predominantly limited to its role in muscle and the nervous system (14). However, numerous studies have demonstrated that chromosome translocation in NSCLC may result in abnormally high expression of MEF2D and promote the formation and development of NSCLC (15). Meanwhile, MEF2D may play an important role in the NSCLC formative process (13). In the present study, we investigated the function of miR-1244 in cisplatin-treated NSCLC.

\section{Materials and methods}

Patient samples and follow-up. In total, 83 fresh tissue samples from cisplatin-induced NSCLC patients and 49 fresh tissue samples from non-cisplatin-induced NSCLC patients were collected at the Department of Thoracic Surgery of the Tumor Hospital of Yunnan Province (The Third Affiliated Hospital of Kunming Medical University) from April 2006 to December 2006. The present study was performed with all the patients written informed consent and in accordance to the procedures approved by the Ethics Review Board at The Third Affiliated Hospital of Kunming Medical University. All patients underwent surgery, and subsequently received cisplatin or no treatment, and were monitored by chest, abdominal and pelvic computed tomography (CT). The overall survival (OS) time was assessed between surgery and cisplatin treatment.

Quantitative real-time RT-PCR ( $q R T-P C R)$. Total RNAs were extracted from the NSCLC tissues using TRIzol reagent (Invitrogen, Carlsbad, CA, USA) according to the manufacturer's instructions. Total RNA $(2 \mu \mathrm{g})$ was reversely-transcribed to cDNA using a First-Strand cDNA synthesis kit (OriGene Technologies, MD, USA). qRT-PCR was conducted using SYBR Premix Taq (CWbio, Beijing, China) on a 7500 Fast PCR instrument (Applied Biosystems, Carlsbad, CA, USA). The relative miR-1244 expression was normalized to U6, which was calculated using the $2^{-\Delta \Delta \mathrm{Ct}}$ method.

Cell culture and transfection. The human NSCLC cell lines A549 and NCI-H522 were purchased from the Shanghai Cell Bank of the Chinese Academy of Sciences, and cultured in RPMI-1640 medium supplemented with 10\% fetal bovine serum (FBS; both from Gibco, Rockville, MD, USA), $100 \mathrm{U} / \mathrm{ml}$ penicillin $\mathrm{G}$ and $100 \mathrm{mg} / \mathrm{ml}$ streptomycin sulfate at $37^{\circ} \mathrm{C}$ in a humidified 5\% $\mathrm{CO}_{2}$ atmosphere. miR-1244 mimics, si-MEF2D or the negative control were constructed by Sangon Biological Engineering Co., Ltd. (Shanghai, China). miR-1244 mimics, si-MEF2D (30 $\mathrm{nM})$ and the negative control $(30 \mathrm{nM})$ were transfected into cells with Lipofectamine ${ }^{\mathrm{TM}} 2000$ (Invitrogen).

MTT and LDH assays. For the MTT assay, transfected A549 and $\mathrm{H} 522$ cells were seeded $\left(2.5 \times 10^{3}\right.$ cells/well $)$ in 96-well plates and allowed to attach overnight. A549 and H522 cells were cultured with $10 \mu \mathrm{M}$ of cisplatin for $0,24,48$ and $72 \mathrm{~h}$.
MTT (20 $\mu 1$ at $5 \mathrm{mg} / \mathrm{ml}$; Sigma-Aldrich, St. Louis, MO, USA) was added to the medium after treatment at $37^{\circ} \mathrm{C}$ for $4 \mathrm{~h}$. The supernatant was aspirated, and $150 \mu \mathrm{l}$ of dimethyl sulfoxide (DMSO) was added to each well to dissolve the precipitate at $37^{\circ} \mathrm{C}$ for $20 \mathrm{~min}$. The absorbance at $490 \mathrm{~nm}$ was assessed using a microplate reader (Molecular Devices, Sunnyvale, CA, USA).

For the LDH assay, transfected A549 and H522 cells were seeded $\left(2.5 \times 10^{3}\right.$ cells/well $)$ in 96-well plates and allowed to attach overnight. A549 and H522 cells were cultured with $10 \mu \mathrm{M}$ of cisplatin for $48 \mathrm{~h}$. The supernatant was aspirated and $150 \mu \mathrm{l}$ of LDH releasing reagent was added into each well at $37^{\circ} \mathrm{C}$ for $1 \mathrm{~h}$. The absorbance at $490 \mathrm{~nm}$ was assessed using a microplate reader (Molecular Devices).

Flow cytometry. Transfected A549 and H522 cells were seeded $\left(5 \times 10^{6}\right.$ cells/well) in 6-well plates and allowed to attach overnight. A549 and H522 cells were cultured with $10 \mu \mathrm{M}$ of cisplatin for $48 \mathrm{~h}$. The cells were then stained with an Annexin V-FITC and propidium iodide (PI) apoptosis detection kit (MultiSciences Biotech Co., Ltd., Hangzhou, China) at $4^{\circ} \mathrm{C}$ for $20 \mathrm{~min}$ in the dark. Cell apoptosis was assessed on an LSR2 upgraded flow cytometer (BD Biosciences, San Jose, CA, USA).

Western blotting. Transfected A549 and H522 cells were seeded $\left(5 \times 10^{6}\right.$ cells/well) in 6-well plates and allowed to attach overnight. A549 and H522 cells were cultured with $10 \mu \mathrm{M}$ of cisplatin for $48 \mathrm{~h}$. Then, cells were harvested at 3,000 $\mathrm{x}$ g for 5 min and lysed in RIPA buffer in the presence of protease inhibitors (Roche, Mannheim, Germany) at $4^{\circ} \mathrm{C}$ for $30 \mathrm{~min}$. The protein concentration in the supernatants was quantified using the Enhanced BCA Protein Assay kit (Beyotime Biotechnology, Shanghai, China). Proteins (50-80 $\mu \mathrm{g}$ ) were separated using $8-12 \%$ SDS-PAGE, and then transferred to polyvinylidene fluoride (PVDF) membranes (Millipore, Bedford, MA, USA). The membranes were blocked using 5\% non-fat milk in Tris-buffered saline with Tween-20 (TBST) for $1 \mathrm{~h}$ at $37^{\circ} \mathrm{C}$, and incubated with the primary antibodies Bax, MEF2D, cyclin D1, p53 (all from Santa Cruz Biotechnology, Santa Cruz, CA, USA) and GAPDH overnight at $4^{\circ} \mathrm{C}$. Subsequently the membranes were incubated with anti-rabbit horseradish peroxidase-conjugated secondary antibodies (Santa Cruz Biotechnology) and developed using an enhanced chemiluminescence (ECL) detection system (Invitrogen-Life Technologies, Carlsbad, CA, USA).

Statistical analysis. The values in the present study are expressed as the means $\pm \mathrm{SD}$, and were analyzed by $\mathrm{t}$-test or one-way ANOVA. Data were analyzed using SPSS software 19.0 for Windows (SPSS, Inc., Chicago, IL, USA). P $<0.05$ was considered to indicate a statistically significant result.

\section{Results}

Expression of miR-1244 in cisplatin-treated NSCLC. Initially, we surveyed the expression of miR-1244 in cisplatin-treated or non-cisplatin-treated NSCLC tissues and cell lines. The miR-1244 expression of cisplatin-treated NSCLC patient tissues was lower than that of non-cisplatin-treated NSCLC 

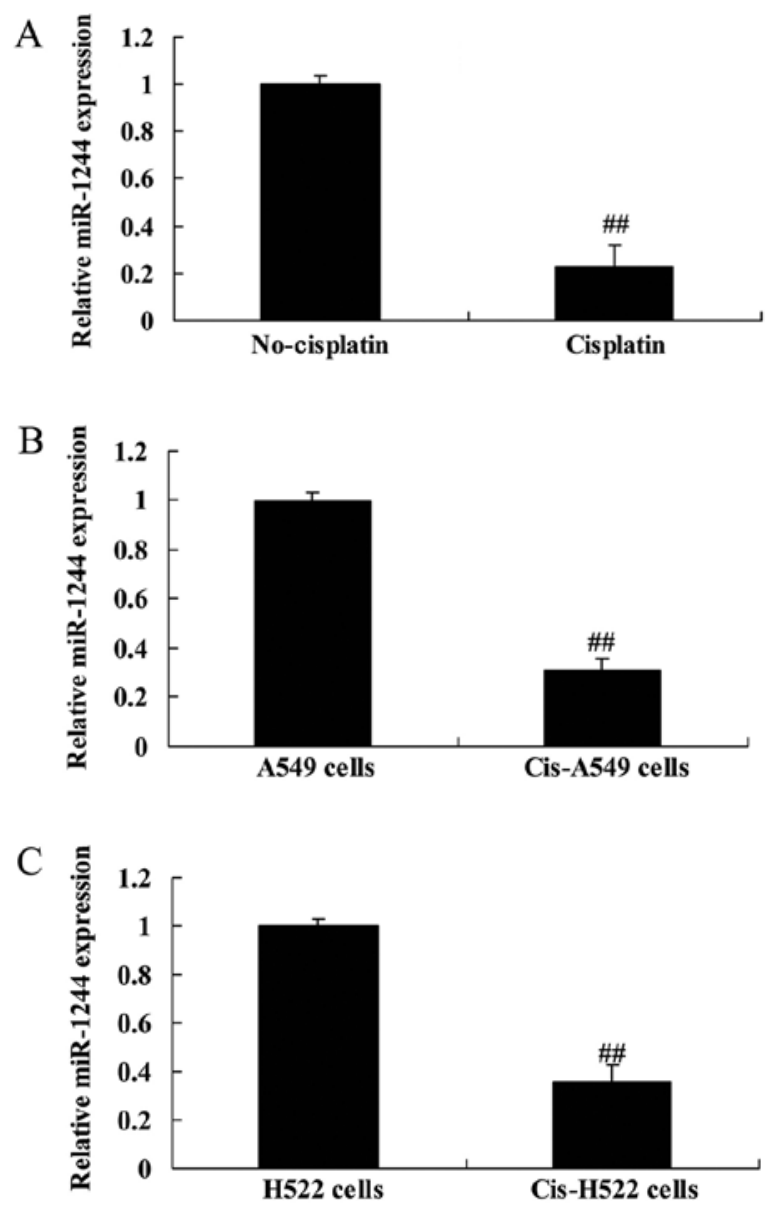

Figure 1. Expression of miR-1244 in cisplatin-induced NSCLC. Expression of miR-1244 in cisplatin or non-induced (A) NSCLC patient tissues and (B) A549 and (C) H522 cell lines; ${ }^{\# \#}$ p $<0.01$ vs. the miR-NC group. NSCLC, non-small cell lung cancer.

patients (Fig. 1A). Consistently, the miR-1244 expression of cisplatin-treated A549 and NCI-H522 cells was also lower than those of the control A549 and NCI-H522 cells (Fig. 1B and C).

Overall survival (OS) of cisplatin-treated NSCLC patients. The OS of cisplatin-treated NSCLC patients was assessed. Among cisplatin-treated NSCLC patients, the OS time of patients with high miR-1244 expression was greater than that of patients with low miR-1244 expression (Fig. 2).

Overexpression of $\mathrm{miR}-1244$ inhibits the growth of cisplatintreated NSCLC cells. MTT and LDH assays were used to investigate the effect of miR-1244 on the growth of cisplatin-treated NSCLC cells. The results demonstrated that the overexpression of miR-1244 significantly inhibited cell proliferation in cisplatin-treated A549 and NCI-H522 cells (Fig. 3A and B). Additionally, overexpression of miR-1244 significantly increased the LDH activity of cisplatin-treated A549 and NCI-H522 cells (Fig. 3C and D).

Overexpression of miR-1244 increases cisplatin-treated NSCLC cell death. The effect of miR-1244 on cisplatin-induced NSCLC cell death was explored using flow cytometry. There was a significant increase in apoptosis in cisplatin-treated A549 and NCI-H522 cells compared with untreated cells (Fig. 4A and B).

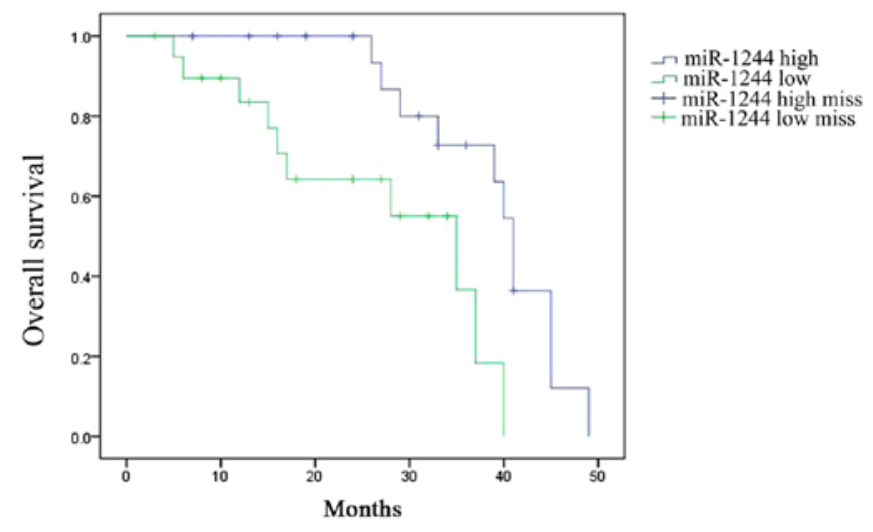

Figure 2. Overall survival of cisplatin-induced NSCLC patients. NSCLC, non-small cell lung cancer.

Overexpression of miR-1244 increases caspase-3 and Bax protein expression in cisplatin-treated NSCLC cells. We next explored the apoptosis-promoting mechanism of miR-1244 in cisplatin-treated NSCLC cells by assessing caspase-3 activity and Bax protein expression. As demonstrated in Fig. 5A and B, increased caspase-3 activity was observed in cisplatin-treated A549 and NCI-H522 cells compared with untreated cells. Furthermore, the induction of Bax protein expression was observed in cisplatin-treated A549 and NCI-H522 cells (Fig. 5C and D and $\mathrm{H}$ and I, respectively).

Overexpression of miR-1244 decreases MEF2D protein expression in cisplatin-treated NSCLC cells. To study the role of MEF2D in the effect of miR-1244 on cisplatin-treated NSCLC, miR-1244 mimics were transfected into cisplatintreated A549 and NCI-H522 cells. As shown in Fig. 5D and E and $\mathrm{H}$ and $\mathrm{J}$, respectively, overexpression of $\mathrm{miR}-1244$ significantly suppressed MEF2D protein expression in cisplatin-treated A549 and NCI-H522 cells.

Overexpression of miR-1244 suppresses cyclin D1 protein expression in cisplatin-treated NSCLC cells. Cyclin D1 protein expression levels were evaluated to assess the apoptosis-promoting mechanism of miR-1244 in cisplatin-treated NSCLC cells. Fig. 5D, F, H and K demonstrate that overexpression of miR-1244 significantly suppressed cyclin D1 protein expression in cisplatin-treated A549 and NCI-H522 cells.

Overexpression of miR-1244 increases p53 protein expression in cisplatin-treated NSCLC cells. p53 protein expression was assessed in cisplatin-treated NSCLC cells in which miR-1244 was overexpressed. Overexpression of miR-1244 significantly induced p53 protein expression in cisplatin-treated A549 and NCI-H522 cells (Fig. 5D, G, H and L).

MEF2D knockdown inhibits MEF2D protein expression in cisplatin-treated NSCLC cells following overexpression of $m i R-1244$. In order to further confirm the role of MEF2D in the effect of miR-1244 on cisplatin-treated NSCLC cells, si-MEF2D was transfected into cisplatin-treated A549 and NCI-H522 cells following overexpression of miR-1244. si-MEF2D effectively inhibited MEF2D protein expression in cisplatin-treated miR-1244-overexpressing A549 

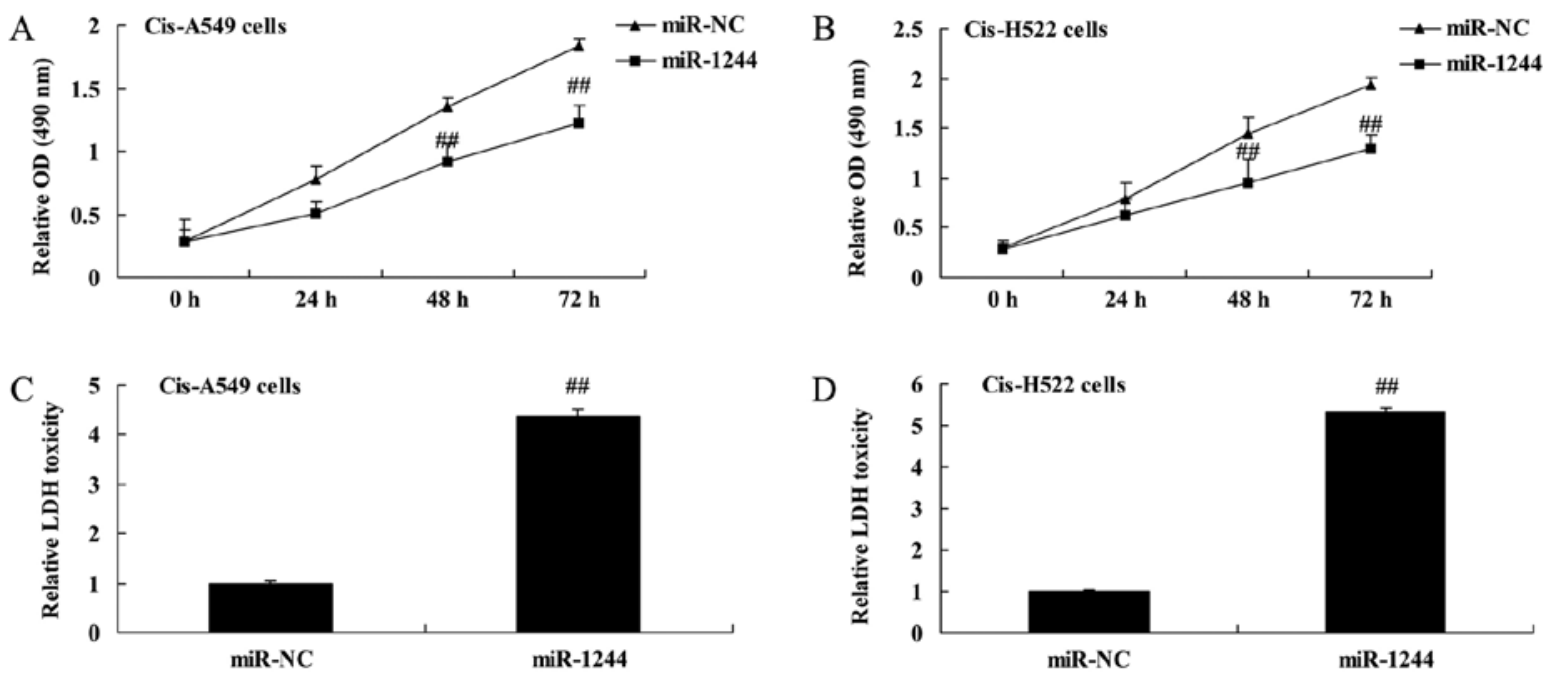

Figure 3. Overexpression of miR-1244 suppresses cisplatin-induced NSCLC cell growth. Overexpression of miR-1244 suppresses (A) cisplatin-induced A549 and (B) H522 cell proliferation as determined using an MTT assay, and LDH toxicity in (C) A549 and (D) H522 cells. miR-NC, miRNA-negative control; miR-1244, miRNA-1244; ${ }^{\# \#} \mathrm{p}<0.01$ vs. the miR-NC. NSCLC, non-small cell lung cancer.
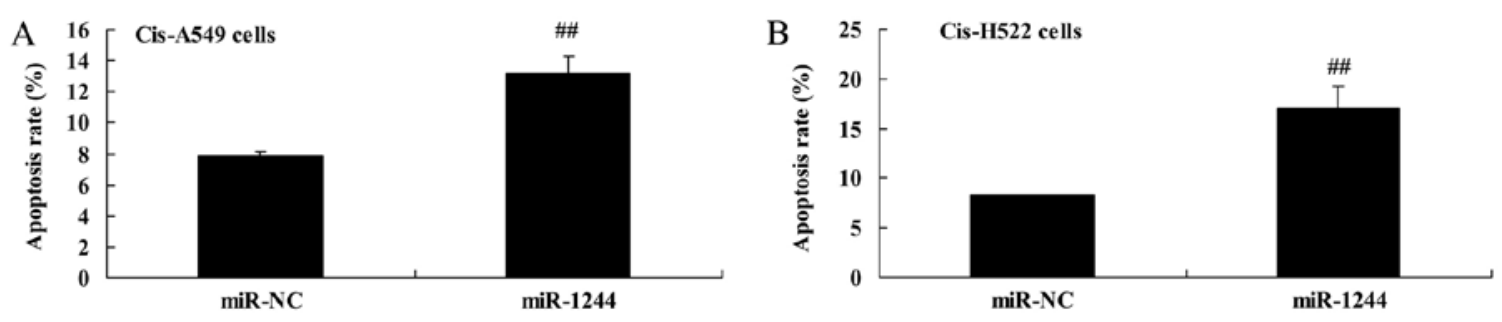

Figure 4. Overexpression of miR-1244 promotes cisplatin-induced NSCLC cell death. Overexpression of miR-1244 promotes cisplatin-induced (A) A549 and (B) H522 cell death. miR-NC, miRNA-negative control; miR-1244, miRNA-1244 group; \# ${ }^{\#}$ $<0.01$ vs. the miR-NC group. NSCLC, non-small cell lung cancer.

and NCI-H522 cells compared with the negative control group (Fig. 6A and B and F and G, respectively).

Inhibition of MEF2D decreases cisplatin-treated NSCLC cell growth following overexpression of miR-1244. The effects of MEF2D inhibition on the growth of cisplatin-treated miR-1244-overexpressing NSCLC cells were next investigated. Fig. 7 shows that the inhibition of MEF2D significantly inhibited cell proliferation and increased LDH activity of cisplatin-treated A549 and NCI-H522 cells following overexpression of miR-1244, compared with the negative control group in which MEF2D was not inhibited.

Inhibition of MEF $2 D$ promotes cisplatin-treated NSCLC cell death following overexpression of miR-1244. We further explored the effects of MEF2D inhibition on cisplatin-treated NSCLC cell death following overexpression of miR-1244. As presented in Fig. 8, the inhibition of MEF2D significantly induced apoptosis of cisplatin-treated A549 and NCI-H522 cells following overexpression of miR-1244, compared with the negative control group in which MEF2D was not inhibited.

Inhibition of MEF2D promotes caspase- 3 and Bax protein expression in cisplatin-treated NSCLC cells following overexpression of miR-1244. The inhibition of MEF2D significantly promoted caspase- 3 activity in cisplatin-treated
A549 and NCI-H522 cells following overexpression of miR-1244, compared with the negative control group (Fig. 9). Furthermore, as shown in Fig. $6 \mathrm{~B}$ and $\mathrm{C}$ and $\mathrm{G}$ and $\mathrm{H}$, respectively, the inhibition of MEF2D significantly increased Bax protein expression in cisplatin-treated A549 and NCI-H522 cells following overexpression of miR-1244 compared with the cells in which MEF2D was not inhibited.

Inhibition of MEF2D suppresses cyclin D1 protein expression in cisplatin-treated NSCLC cells following overexpression of miR-1244. As shown Fig. 6B, D, G and I, in cisplatin-treated miR-1244-overexpressing A549 and NCI-H522 cells, inhibition of MEF2D significantly suppressed cyclin D1 protein expression compared with the negative control group.

Inhibition of MEF2D increases p53 protein expression in cisplatin-treated NSCLC cells following overexpression of $m i R-1244$. As shown in Fig. 6B, E, G and J, in cisplatin-treated miR-1244-overexpressing A549 and NCI-H522 cells, the inhibition of MEF2D significantly induced $\mathrm{p} 53$ protein expression compared with the negative control group.

\section{Discussion}

NSCLC is one of the most common malignant tumors, and accounts for $80-85 \%$ of all lung cancers (3). It has a high 
A

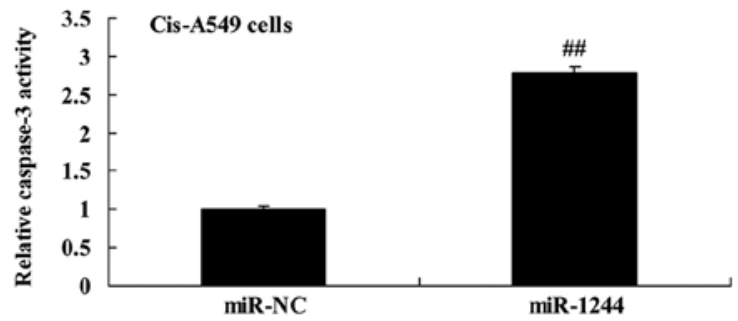

C

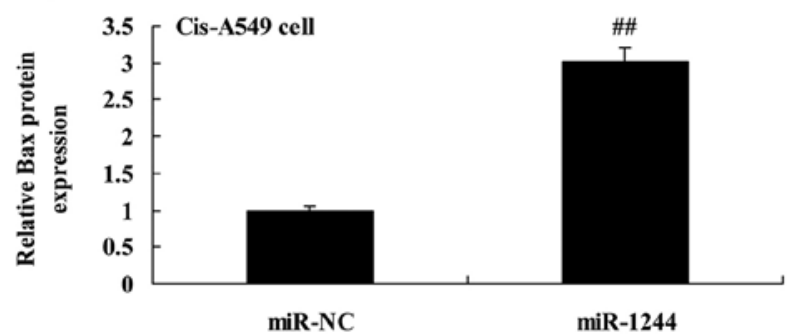

E

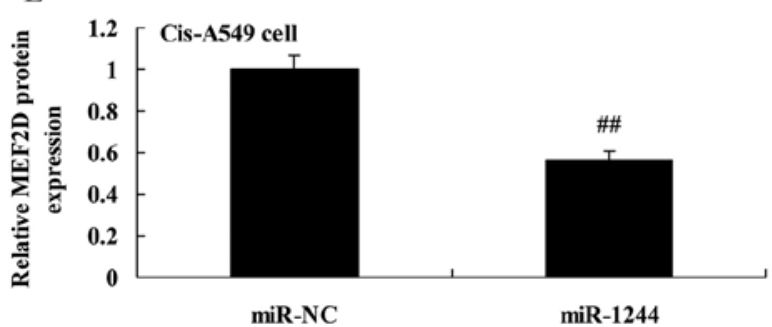

G

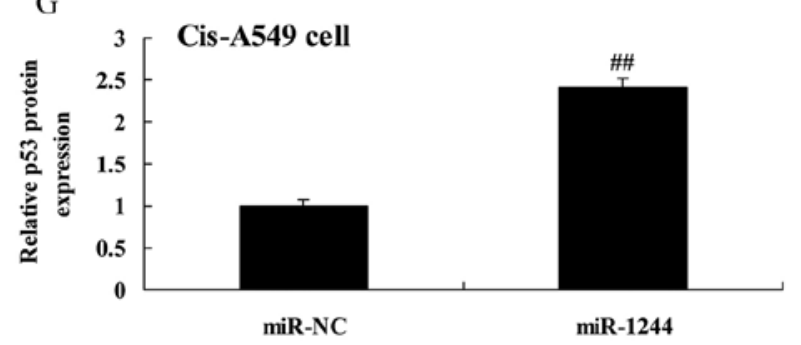

I

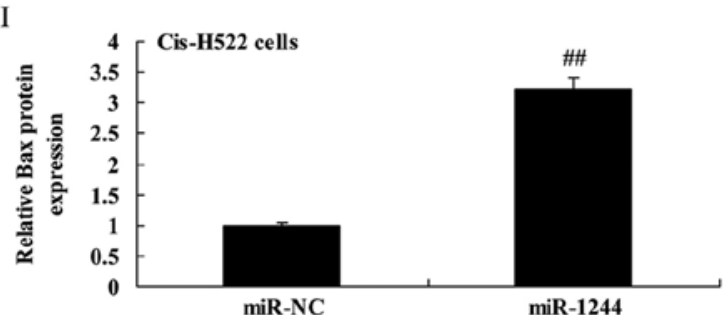

K

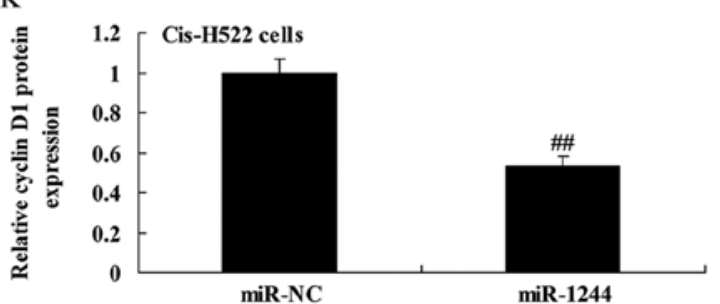

B

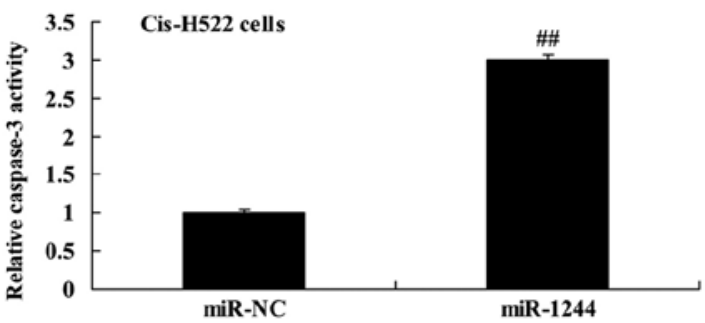

D

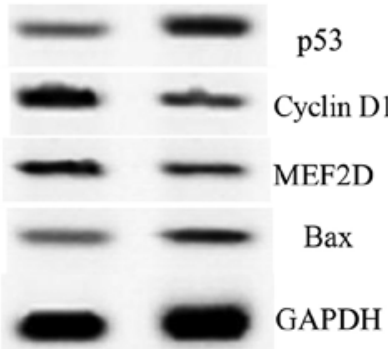

miR-NC miR-1244

F

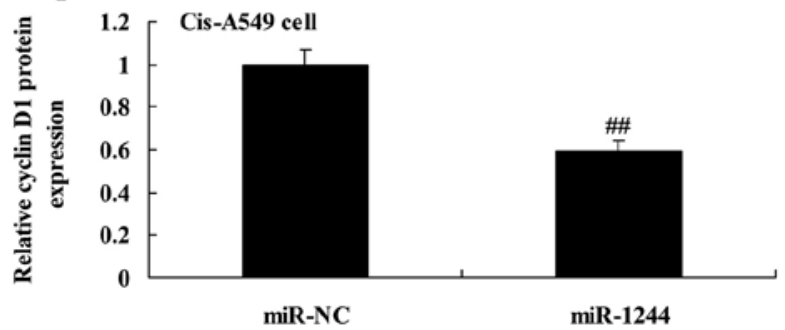

$\mathrm{H}$

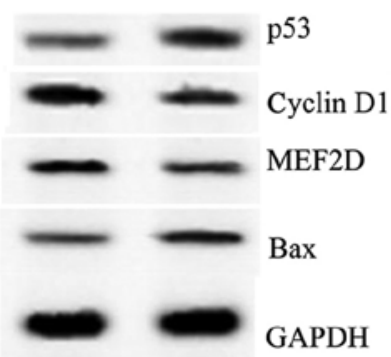

Cis-H522 cells

miR-NC miR-1244

J

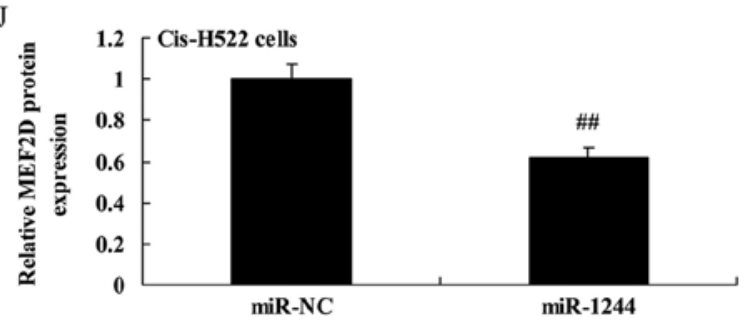

L

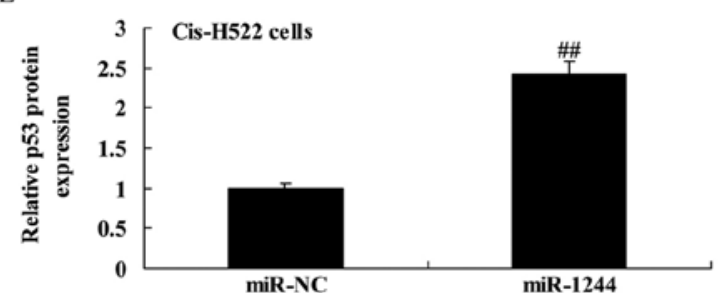

Figure 5. Overexpression of miR-1244 affects caspase-3, Bax, MEF2D, cyclin D1 and p53 protein expression in cisplatin-induced NSCLC cells. (A and B) Overexpression of miR-1244 affects caspase-3 and (C, E-G and I-L) Bax, MEF2D, cyclin D1 and p53 protein expression using statistical analysis. (D and H) Western blot analysis of Bax, MEF2D, cyclin D1 and p53 protein expression in cisplatin-induced A549 and H522 cells. miR-NC, miRNAnegative control; miR-1244, miRNA-1244 group; ${ }^{\# \#}$ p $<0.01$ vs. the miR-NC group. MEF2D, myocyte enhancer factor 2D; NSCLC, non-small cell lung cancer. 
A

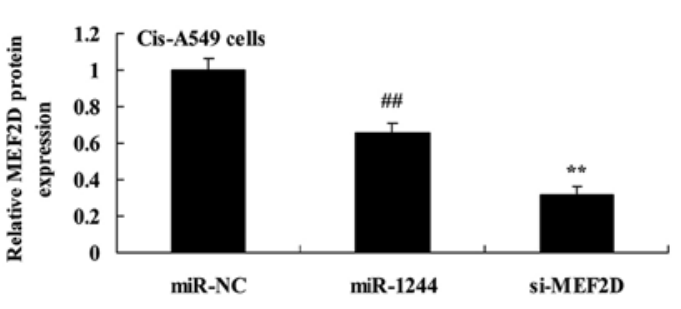

$\mathrm{C}$

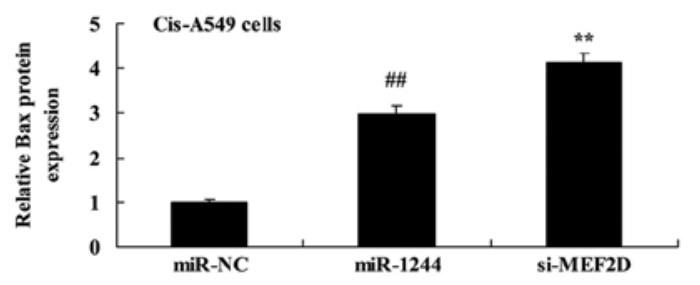

$\mathrm{E}$

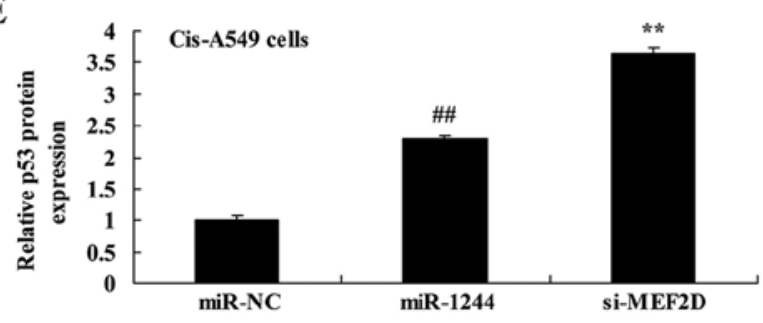

G

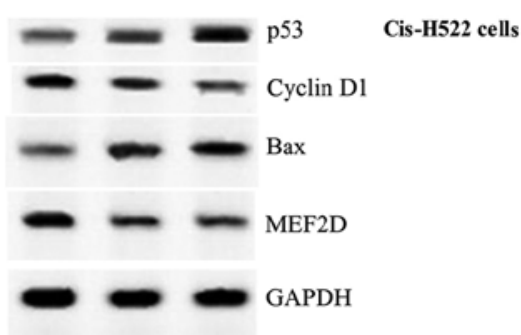

miR-NC miR-1244 si-MEF2D

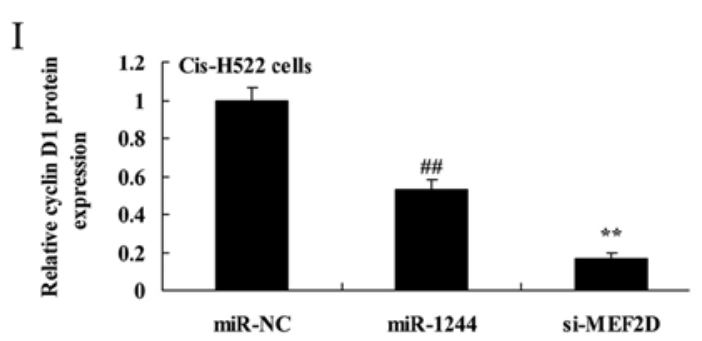

B

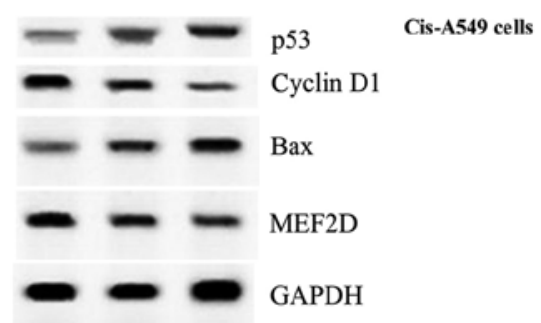

miR-NC miR-1244 si-MEF2D

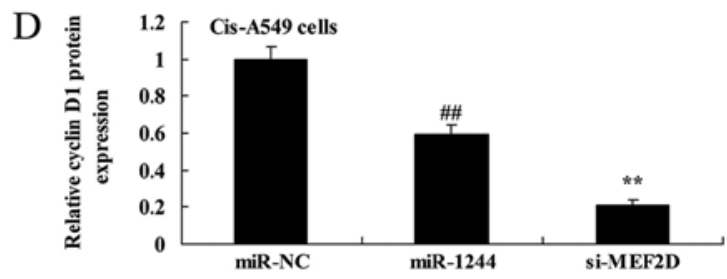

F

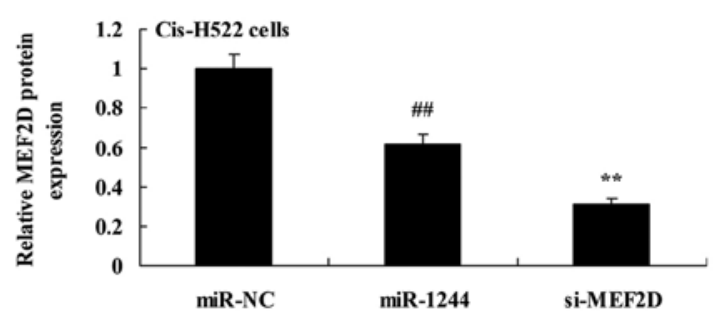

$\mathrm{H}$
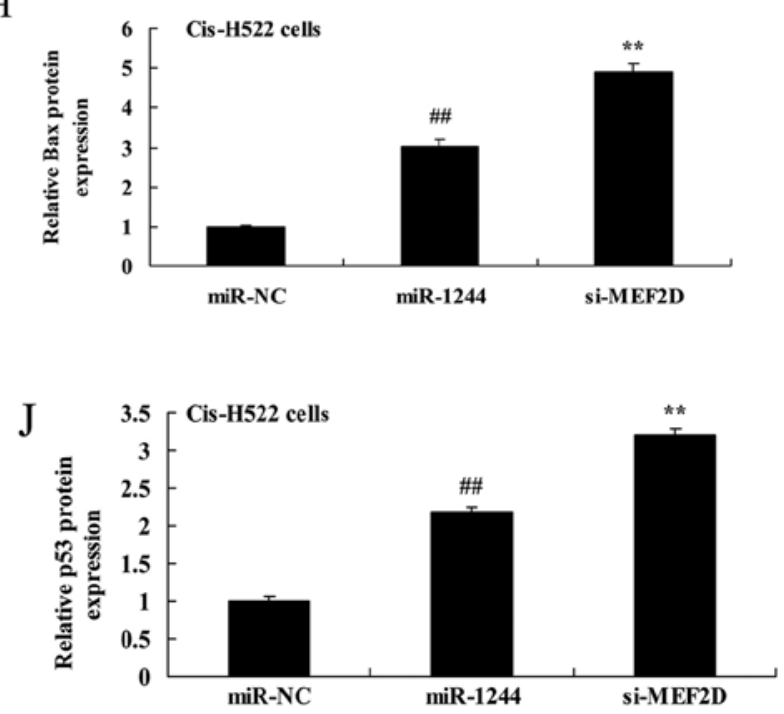

Figure 6. Inhibition of MEF2D affects MEF2D, Bax, cyclin D1 and p53 protein expression in cisplatin-induced NSCLC cells following overexpression of miR-1244. (A, C-F and H-J) Inhibition of MEF2D affects MEF2D, Bax, cyclin D1 and p53 protein expression using statistical analysis. (B and G) Western blot analysis of MEF2D protein expression in cisplatin-induced NSCLC following overexpression of miR-1244. miR-NC, miRNA-negative control; miR-1244, miRNA-1244; si-MEF2D, si-MEF2D group; ${ }^{\# \#}$ p<0.01 vs. the miR-NC group; ${ }^{* *}$ p $<0.01$ vs. the miRNA-1244 group. MEF2D, myocyte enhancer factor 2D; NSCLC, non-small cell lung cancer.

mortality rate and presents a significant threat to human life and health worldwide, with $\sim 1.1$ million patients each year succumbing to NSCLC (16). Despite extensive research efforts, the overall effects of NSCLC treatment remain unsatisfactory. In recent years, NSCLC has exhibited a rising morbidity rate. Furthermore, the pathogenesis of NSCLC has not been established $(17,18)$. Currently, it is believed that cell cycle control abnormalities are associated with the cancerous transformation of cells; malignant tumors may have a dysregulated cell cycle (17). In the present study, we observed that the expression levels of miR-1244 in the cisplatin-treated A549 and NCI-H522 cells were lower than those of the untreated A549 and NCI-H522 cells, and that the OS time of the cisplatin-treated NSCLC patients with high miR-1244 expression was greater than the 

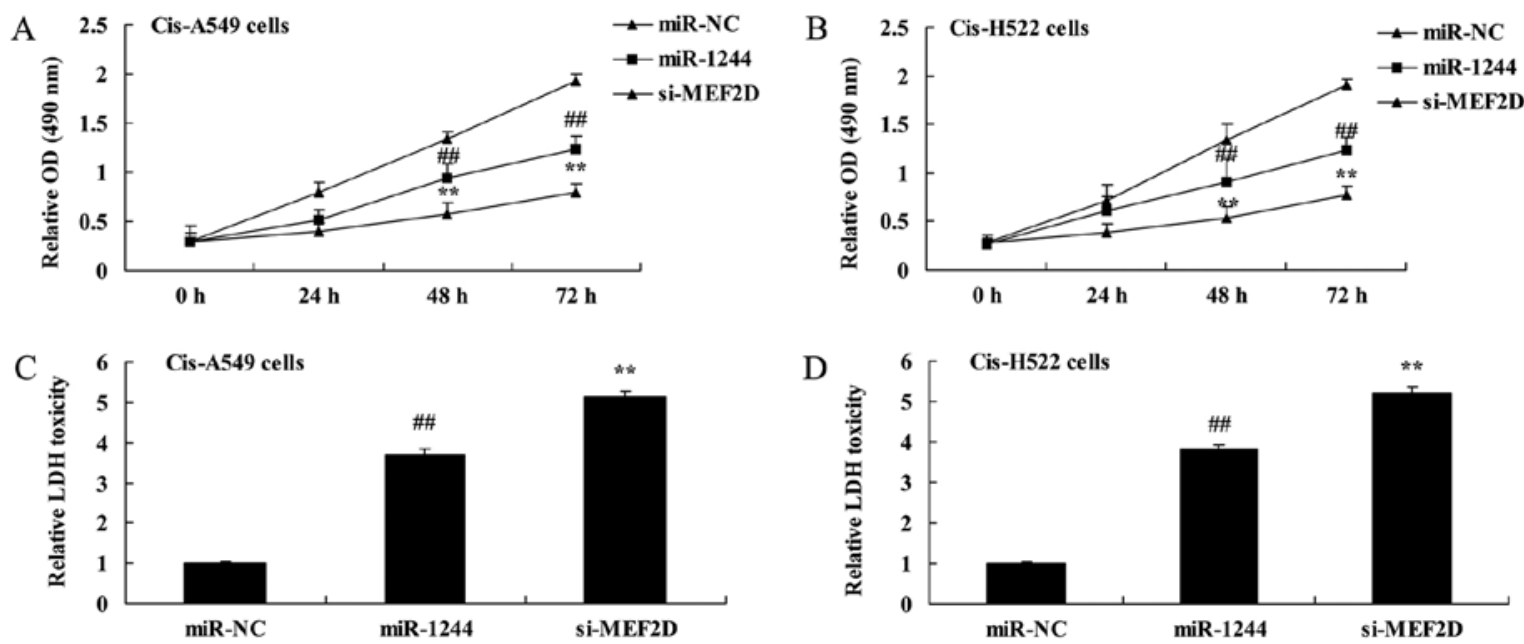

Figure 7. Inhibition of MEF2D affects cisplatin-induced NSCLC growth following overexpression of miR-1244. Inhibition of miR-1244 affects cisplatin-induced (A) A549 and (B) H522 cell proliferation using MTT assay, and LDH toxicity in (C) A549 and (D) H522 cells. miR-NC, miRNA-negative control; miR-1244, miRNA-1244; si-MEF2D, si-MEF2D group; ${ }^{\# \#}$ p $<0.01$ vs. the miR-NC group; ${ }^{* *} \mathrm{p}<0.01$ vs. the miRNA-1244 group. MEF2D, myocyte enhancer factor 2D; NSCLC, non-small cell lung cancer.
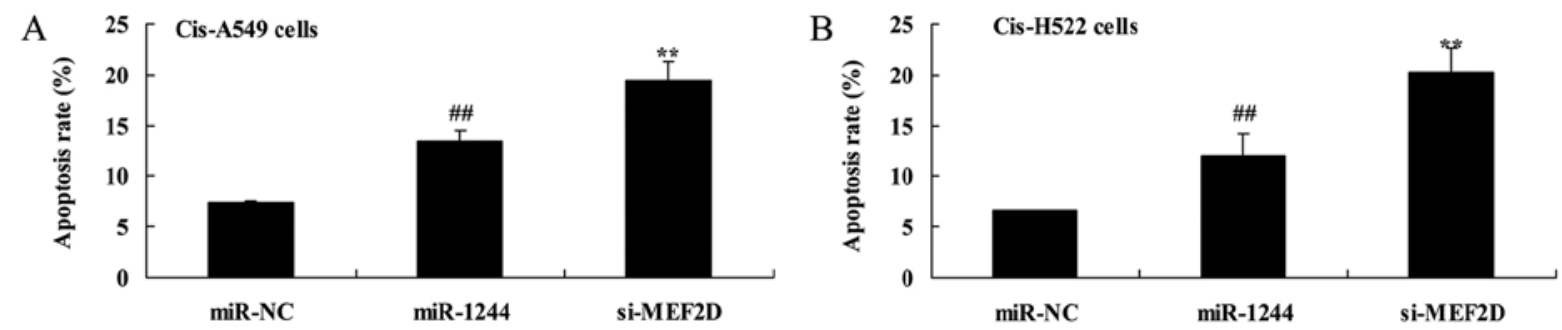

Figure 8. Inhibition of MEF2D affects cisplatin-induced NSCLC cell death following overexpression of miR-1244. Inhibition of miR-1244 affects cisplatin-induced (A) A549 and (B) H522 cell death. miR-NC, miRNA-negative control; miR-1244, miRNA-1244; si-MEF2D, si-MEF2D group; ${ }^{\# \#}$ p $<0.01$ vs. the miR-NC group; ${ }^{* *}$ p $<0.01$ vs. the miRNA-1244 group. MEF2D, myocyte enhancer factor 2D; NSCLC, non-small cell lung cancer.
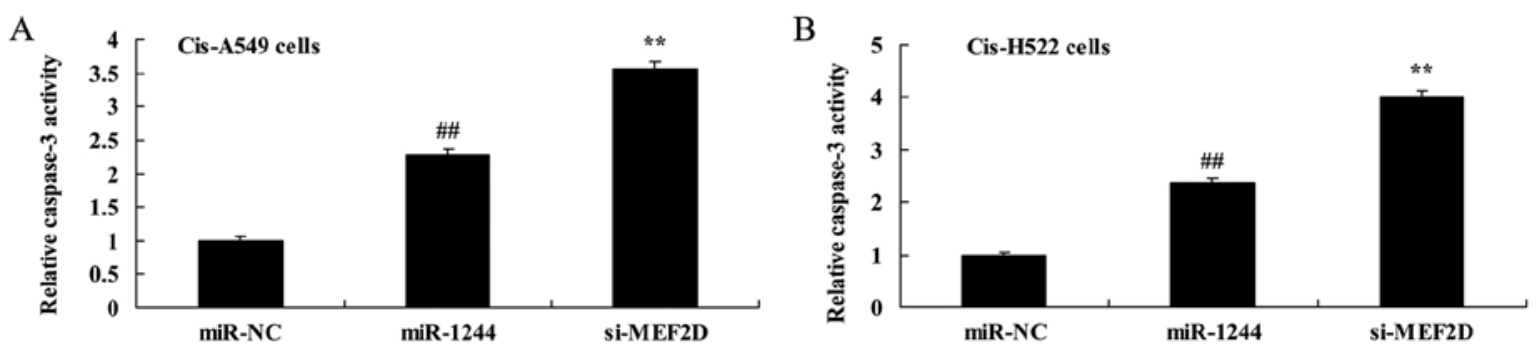

Figure 9. Inhibition of MEF2D affects caspase-3 expression in cisplatin-induced NSCLC following overexpression of miR-1244. (A and B) Inhibition of miR-1244 affects caspase-3 in cisplatin-induced A549 and H522 cells. miR-NC, miRNA-negative control; miR-1244, miRNA-1244; si-MEF2D, si-MEF2D group; ${ }^{\# \#} \mathrm{p}<0.01$ vs. the miR-NC group; ${ }^{*} \mathrm{p}<0.01$ vs. the miRNA-1244 group. MEF2D, myocyte enhancer factor 2D; NSCLC, non-small cell lung cancer.

patients with low miR-1244 expression. Thus, miR-1244 may play an essential role in NSCLC development.

NSCLC is the most common cause of death resulting from a malignant tumor (19). Although diagnosis and treatment methods are improving constantly, the prognosis of patients with NSCLC remains poor (19). At present, clinical and pathological staging of NSCLC is the ideal method to evaluate NSCLC prognosis and select optimal treatment. The identification of miRNAs in studies of tumors and other diseases has led to much research into the alterations in miRNA expression associated with NSCLC (20). In tumor subtype classification, abnormal miRNA expression profiles have been detected (8).
Such reports are few in number at present; however, many miRNA alterations are being discovered constantly. The integration of miRNA expression analysis with NSCLC staging may improve the diagnosis and prognosis of NSCLC, and it may aid in the individual treatment of NSCLC (11). To the best of our knowledge, the present study is the first to show that the overexpression of miR-1244 can suppress cell viability, increase LDH toxicity, induce apoptosis, and promote caspase-3 activity and Bax protein expression in cisplatin-treated A549 and NCI-H522 cells. Notably, for the first time, we established the link between miR-1244 expression and cisplatin-treated NSCLC cell growth. 


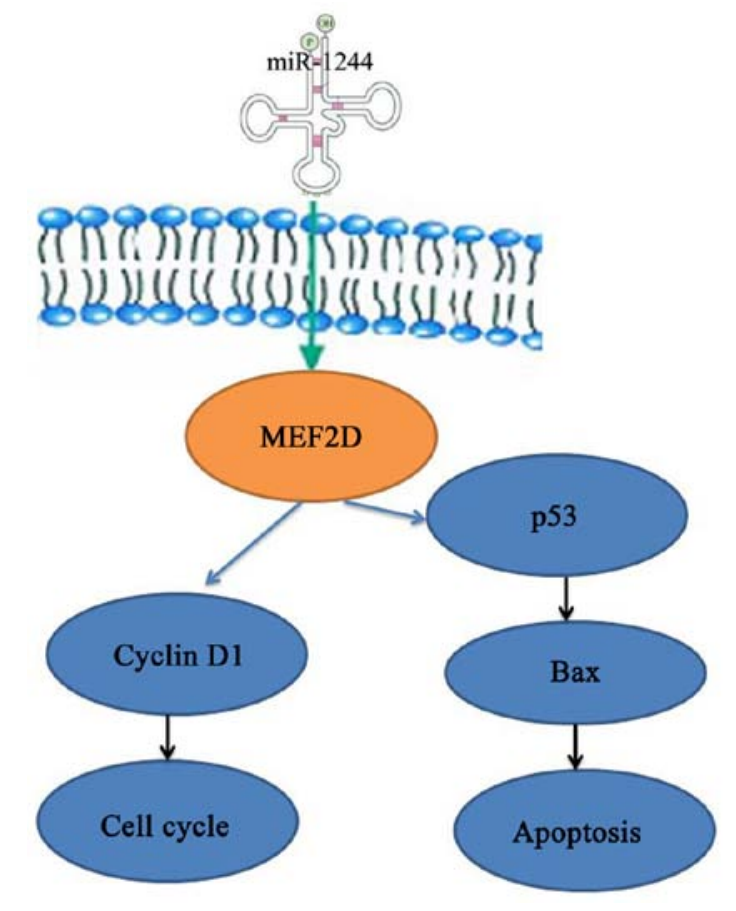

Figure 10. Effect of miR-1244 on cisplatin-induced NSCLC via MEF2D expression. MEF2D, myocyte enhancerfactor 2D; NSCLC, non-small cell lung cancer.

MEF2D, a member of the myocyte enhancer factor 2 family of transcription factors, has been shown to be expressed in NSCLC cells and to increase the proliferation rate of cells by increasing G2/M transition (14). MEF2D is a target of miR-122 (13). Thus, MEF2D may be a potential target for use in cancer treatments. This is consistent with our observation that the overexpression of miR-1244 significantly suppressed MEF2D protein expression in cisplatin-treated A549 and NCI-H522 cells. These results indicated that miR-1244/MEF2D play an important role in the development of cisplatin resistance in NSCLC.

The cell cycle refers to the entire process between cell fission and the end of the next mitosis process, in which a parent cell is divided into two daughter cells (22). The cell cycle is composed of two main stages: interphase, which can be further divided into gap 1 (G1), DNA synthesis (S), and gap 2 (G2) phases (23); and mitosis, which is comprised of prophase, metaphase, anaphase and telophase, and is responsible for the division of a cell's genetic material prior to cytokinesis (24). The mechanisms involved in the control of the cell cycle have become an important area of research (23). In the present study, it was demonstrated that the overexpression of miR-1244 significantly suppressed cyclin D1 protein expression in cisplatin-treated A549 and NCI-H522 cells, indicating that miR-1244/MEF2D may target cyclin D1, and that miR-1244 exerts its antitumor effect by suppressing the development of cisplatin resistance in NSCLC. Our study indicated that oncogenic MEF2D is also a target of miR-1244, and at least partially, miR-1244 exerts its antitumor effect by suppressing MEF2D expression.

Cyclins are proteins that contain a conserved cyclin box structure, and they participate in the regulation of the cell cycle (25). Cyclins and cyclin-dependent kinases form complexes that play roles in regulating different phases of cell division and allowing transitions at each checkpoint. Cyclin D is a subtype of the cyclin family (26). Cyclins B1 and D1 are involved in cell division and are important regulatory factors in the growth phases. The cyclin D family may play a role in cell cycle control and DNA repair and participate in apoptosis (27). In different types of tissue and tumor cells, its expression and biological functions vary (28).

In the present study, we also found that the overexpression of miR-1244 significantly induced p53 protein expression in cisplatin-treated A549 and NCI-H522 cells. Therefore, our study adds p53 to the list of miR-1244/MEF2D-regulated molecules that are involved in the development of cisplatin resistance in NSCLC. Further studies are required to explore the underlying mechanisms of miR-1244/MEF2D in cyclin D1-p53 signaling in cisplatin-treated NSCLC.

Finally, to explore the possible regulatory mechanism of MEF2D inhibition in cisplatin resistance in NSCLC, we found that siRNA-mediated knockdown suppressed the protein expression of MEF2D, and was able to decrease cell proliferation, promote caspase- 3 activity, increase p53 and Bax protein expression, and inhibit cyclin D1 protein expression in cisplatin-treated A549 and NCI-H522 cells following overexpression of miR-1244. In conclusion, our study demonstrates that a miR-1244/MEF2D/cyclin D1-p53 signaling network contributes to the regulation of NSCLC cell growth, and provides a novel potential molecular target for future NSCLC cancer therapy (Fig. 10).

\section{Acknowledgements}

The present study was supported by The Key Program of The National Natural Science Foundation-Yunnan United Foundation (U1202224).

\section{References}

1. Herbst RS, Baas P, Kim DW, Felip E, Pérez-Gracia JL, Han JY, Molina J, Kim JH, Arvis CD, Ahn MJ, et al: Pembrolizumab versus docetaxel for previously treated, PD-L1-positive, advanced non-small-cell lung cancer (KEYNOTE-010): A randomised controlled trial. Lancet 387: 1540-1550, 2016.

2. Singhal N, Vatandoust S and Brown MP: Phase II study evaluating efficacy and safety of everolimus with letrozole for management of advanced (unresectable or metastatic) non-small cell lung cancer after failure of platinum-based treatment: A preliminary analysis of toxicity. Cancer Chemother Pharmacol 75: 325-331, 2015.

3. Langer CJ, Novello S, Park K, Krzakowski M, Karp DD, Mok T, Benner RJ, Scranton JR, Olszanski AJ and Jassem J: Randomized, phase III trial of first-line figitumumab in combination with paclitaxel and carboplatin versus paclitaxel and carboplatin alone in patients with advanced non-small-cell lung cancer. J Clin Oncol 32: 2059-2066, 2014.

4. Heigener DF, Pereira JR, Felip E, Mazal J, Manzyuk L, Tan EH, Merimsky O, Sarholz B, Esser R and Gatzemeier U: Weekly and every 2 weeks cetuximab maintenance therapy after platinumbased chemotherapy plus cetuximab as first-line treatment for non-small cell lung cancer: Randomized non-comparative phase IIIb NEXT trial. Target Oncol 10: 255-265, 2015.

5. Pu Q, Huang Y, Lu Y, Peng Y, Zhang J, Feng G, Wang C, Liu L and Dai Y: Tissue-specific and plasma microRNA profiles could be promising biomarkers of histological classification and TNM stage in non-small cell lung cancer. Thorac Cancer 7: 348-354, 2016.

6. Pastorkova Z, Skarda J and Andel J: The role of microRNA in metastatic processes of non-small cell lung carcinoma. Biomed Pap Med Fac Univ Palacky Olomouc Czech Repub 160: 343-357, 2016. 
7. Wang G, Wang R, Strulovici-Barel Y, Salit J, Staudt MR, Ahmed J, Tilley AE, Yee-Levin J, Hollmann C, Harvey BG, et al: Persistence of smoking-induced dysregulation of miRNA expression in the small airway epithelium despite smoking cessation. PLoS One 10: e0120824, 2015.

8. Bianchi F, Nicassio F, Marzi M, Belloni E, Dall'olio V, Bernard L, Pelosi G, Maisonneuve P, Veronesi G and Di Fiore PP: A serum circulating miRNA diagnostic test to identify asymptomatic high-risk individuals with early stage lung cancer. EMBO Mol Med 3: 495-503, 2011

9. Lee JH, Voortman J, Dingemans AM, Voeller DM, Pham T, Wang Y and Giaccone G: MicroRNA expression and clinical outcome of small cell lung cancer. PLoS One 6: e21300, 2011.

10. Kumarswamy R,Mudduluru G,Ceppi P,Muppala S,KozlowskiM Niklinski J, Papotti M and Allgayer H: MicroRNA-30a inhibits epithelial-to-mesenchymal transition by targeting Snail and is downregulated in non-small cell lung cancer. Int J Cancer 130: 2044-2053, 2012.

11. Tibaldi C, D'Incecco A and Lagana A: MicroRNAs and Targeted Therapies in Non-small Cell Lung Cancer: Minireview. Anticancer Agents Med Chem 15: 694-700, 2015.

12. Ma R, Wang C, Wang J, Wang D and Xu J: miRNA-mRNA interaction network in non-small-cell lung cancer.Interdiscip Sci 209-219, 2016

13. Zhao X, Liu M and Li D: Oleanolic acid suppresses the proliferation of lung carcinoma cells by miR-122/Cyclin G1/MEF2D axis. Mol Cell Biochem 400: 1-7, 2015.

14. Song L, Li D, Zhao Y, Gu Y, Zhao D, Li X, Bai X, Sun Y, Zhang X, Sun H, et al: miR-218 suppressed the growth of lung carcinoma by reducing MEF2D expression. Tumour Biol 37: 2891-2900, 2016

15. Zhang R, Zhang Y and Li H: miR-1244/myocyte enhancer factor $2 \mathrm{D}$ regulatory loop contributes to the growth of lung carcinoma. DNA Cell Biol 34: 692-700, 2015.

16. Yao L, Xu S, Xu J, Yang C, Wang J and Sun D: S-1 plus cisplatin with concurrent radiotherapy versus cisplatin alone with concurrent radiotherapy for stage III non-small cell lung cancer: A pilot randomized controlled trial. Radiat Oncol 10: 10, 2015

17. Hattori Y, Satouchi M, Shimada T, Urata Y, Yoneda T, Mori M, Nishimura T, Sunadome H, Kumagai T, Imamura F, et al: A phase 2 study of bevacizumab in combination with carboplatin and paclitaxel in patients with non-squamous non-small-cell lung cancer harboring mutations of epidermal growth factor receptor (EGFR) after failing first-line EGFR-tyrosine kinase inhibitors (HANSHIN Oncology Group 0109). Lung Cancer 87: 136-140, 2015.
18. Mariano C, Bosdet I, Karsan A, Ionescu D, Murray N, Laskin JJ, Zhai Y, Melosky B, Sun S and Ho C: A population-based review of the feasibility of platinum-based combination chemotherapy after tyrosine kinase inhibition in EGFR mutation positive non-small cell lung cancer patients with advanced disease. Lung Cancer 83: 73-77, 2014.

19. Rothschild SI: Epigenetic Therapy in Lung Cancer - Role of microRNAs. Front Oncol 3: 158, 2013

20. Buitrago DH, Patnaik SK, Kadota K, Kannisto E, Jones DR and Adusumilli PS: Small RNA sequencing for profiling microRNAs in long-term preserved formalin-fixed and paraffin-embedded non-small cell lung cancer tumor specimens. PLoS One 10: e0121521, 2015.

21. Kim MK, Kim SC, Kang JI, Hyun JH, Boo HJ, Eun SY, Park DB, Yoo ES, Kang HK and Kang JH: 6-Hydroxydopamine-induced PC12 cell death is mediated by MEF2D down-regulation. Neurochem Res 36: 223-231, 2011.

22. Qiao WL, Hu HY, Shi BW, Zang LJ, Jin W and Lin Q: Lentivirusmediated knockdown of TSP50 suppresses the growth of non-small cell lung cancer cells via G0/G1 phase arrest. Oncol Rep 35: 3409-3418, 2016.

23. Zhang $\mathrm{XS}$, Zhao $\mathrm{C}$, Tang $\mathrm{WZ}, \mathrm{Wu} \mathrm{XJ}$ and Zhao YQ: Gypensapogenin $\mathrm{H}$, a novel dammarane-type triterpene induces cell cycle arrest and apoptosis on prostate cancer cells. Steroids 104: 276-283, 2015.

24. Zhu J, Chen M, Chen N, Ma A, Zhu C, Zhao R, Jiang M, Zhou J, Ye L, Fu H, et al: Glycyrrhetinic acid induces G1-phase cell cycle arrest in human non-small cell lung cancer cells through endoplasmic reticulum stress pathway. Int J Oncol 46: 981-988, 2015.

25. Liao K, Li J and Wang Z: Dihydroartemisinin inhibits cell proliferation via AKT/GSK3 $\beta /$ cyclin D1 pathway and induces apoptosis in A549 lung cancer cells. Int J Clin Exp Pathol 7: 8684-8691, 2014.

26. Li Q, Dong Q and Wang E: Rsf-1 is overexpressed in non-small cell lung cancers and regulates cyclin D1 expression and ERK activity. Biochem Biophys Res Commun 420: 6-10, 2012.

27. Recchia AG, Musti AM, Lanzino M, Panno ML, Turano E, Zumpano R, Belfiore A, Andò S and Maggiolini M: A cross-talk between the androgen receptor and the epidermal growth factor receptor leads to p38MAPK-dependent activation of mTOR and cyclin D1 expression in prostate and lung cancer cells. Int J Biochem Cell Biol 41: 603-614, 2009.

28. Lingfei K, Pingzhang Y, Zhengguo L, Jianhua G and Yaowu Z A study on p16, pRb, cdk4 and cyclin D1 expression in non-small cell lung cancers. Cancer Lett 130: 93-101, 1998. 